\title{
TITLE Stability of Omega-3 Fatty Acids in Fortified Surimi Seafoods during Chilled Storage
}

2 AUTHOR ${ }^{\mathrm{a}}$ Miriam Pérez-Mateos, ${ }^{\mathrm{b}}$ Leon Boyd, and ${ }^{\mathrm{b}} *$ Tyre Lanier

AUTHOR ${ }^{a}$ Instituto del Frío (CSIC), Madrid, Spain, and ${ }^{b}$ North Carolina State University, Department

4 of Food Science, Raleigh, NC 27695-7624, USA

AUTHOR EMAIL ADDRESS * Corresponding author: Leon Boyd North Carolina State University,

6 Department of Food Science (E-mail: leon_boyd@ncsu.edu; (phone 919-513-2259, Fax 919-515-7124)

RECEIVED DATE (to be automatically inserted after your manuscript is accepted if required according

8 to the journal that you are submitting your paper to)

TITLE RUNNING HEAD Stability of omega-3 fatty acids in seafood analogue

10 ABSTRACT Physical, chemical and sensory properties of cooked surimi seafood gels (crab analog) fortified with omega-3 fatty acids (FA) were monitored during chilled storage. Three sources of

12 stabilized omega-3 FA (fish oil concentrate, menhaden oil, and a purified marine oil) were each incorporated into gels to an omega-3 FA content of $1.5 \%$ or $2.5 \%$, wt/wt. Omega-3 FA stability,

14 development of off-flavors, and changes in color and texture were monitored during chilled storage for two months. Gels with fish oil concentrate developed fishy flavor and aroma within 30 days and were eliminated from the study. Gels containing menhaden oil and purified marine oil exhibited little change in sensory properties or oxidation products throughout two months of storage. Relative polyene index values (ratio of polyunsaturated to saturated fatty acids) indicated that the omega-3 FA were stable at both levels of addition. Omega-3 fortified gels were whiter than control gels and gel texture was modify

20 when adding menhaden and purified oil but not significantly affected by level of omega-3 addition. 
Consumption of polyunsaturated fatty acids (PUFA) of the omega-3 series, especially eicosapentenoic

26 acid (EPA) and docosahexaenoic acid (DHA), exerts a strong positive influence on decreasing the risk factors associated with the occurrence of several degenerative diseases including cancer, cardiovascular diseases, rheumatoid arthritis, and other inflammatory conditions (1-2). As the major sources of omega3 FA are fish and other marine-derived food products, the daily dietary intake of omega-3 FA in some populations may be extremely low. The use of fish oils in food fortification has been limited due to the greater tendency of long-chain fatty acids to oxidize, resulting in off flavors and aromas and consequently substantial reduction in the shelf life of products. Despite this concern, increased demand for omega-3 FA in the food supply coupled with the introduction of more refined and stabilized fish oils

34 have generated research efforts to fortify a wider variety of food products with omega-3 FA. These include such products as bread, milk, ice cream and other foods, not usually associated with marine origins (3-6). Successful fortification of such foods with omega-3 FA have ranged from $0.2 \%$ to $1.8 \%$, depending on the kind of food and presence or absence of other flavor masking components (4). For example, in bread, the maximum acceptable level of omega-3 fortification depends on the formulation, particularly the type of flour, as there are major differences in flavor profile of whole wheat flour vs. white flour (5). Acceptance of omega-3 fortified products also depends on the form of PUFA added and the processing variations. Ice creams to which omega-3 FA were added at concentrations of 42 250mg/100g serving (0.25\%) exhibited negligible fish oil flavors using liquid refined fish oil or microencapsulated omega-3’s from microalgae. However fishy flavors and aromas were noted in ice

44 creams using other sources such as a microencapsulated fish oil powder and cyclodextrin-complexed fish oil (7).

46 Because surimi-seafoods are associated with marine sources, are formulated foods, and enjoy wide acceptance in most developed nations, they have become a logical vehicle for increasing the 48 consumption of omega-3 FA without the need for dietary supplements in pill or capsule form (8). .J. Agric. Food Chem. 2004;52(26):7944-7949 
The objectives of this research were to fortify a surimi-based crab analog to an omega-3 content

50 equivalent to or greater than that of natural salmon fillets (ca.1.5\%) (9-10) such that product quality and nutritional value were not compromised during a period of chilled storage representative of commercial 52 marketing practices.

\section{MATERIALS AND METHODS}

Frozen surimi commercially prepared from Alaska Pollock (Theragra chalcogramma) containing 4 \% sorbitol, $4 \%$ sucrose and $0.15 \%$ sodium tripolyphosphate and $0.15 \%$ tetra sodium pyrophosphate was obtained from Trident Seafoods Corporation (Seattle, WA). Frozen blocks of surimi (10 Kg each) that had been held at $-20^{\circ} \mathrm{C}$ were impact-shattered, portioned into $1 \mathrm{Kg}$ units, vacuum-packaged in oxygen impermeable bags (CN-590 cook-in material bags, Cryovac, Duncan, SC), and stored at $-20{ }^{\circ} \mathrm{C}$ until needed. Moisture content of surimi was determined to be $76.4 \%$ (11).

Preparation of surimi paste. Control gels to which no oil was added consisted of added salt and water to levels of $1.5 \%(\mathrm{w} / \mathrm{w})$ and $75 \%(\mathrm{w} / \mathrm{w})$, respectively. Other additives were $8 \%$ potato starch (Pencook 20 ${ }^{\mathrm{TM}}$, Penford Food Ingredients Co., Englewood, NJ); 2\% egg white powder (McWaldbaum, Wakefield,

64 NE); 1.0\% sorbitol (sorbitol 70/20, Roquette America, Inc., Keokuk, IA); 1.0 \% sucrose; and 3.7\% (w/w) of crab flavor and aroma additives supplied by Activ International (Seattle, WA). This

66 formulation closely imitates that of most commercial crab analog products being marketed.

In treatments containing added omega-3 FA oils, the amount of water was substituted by oil. Three 68 sources of omega-3 FA were used: a fish oil concentrate (20/50 TG ${ }^{\mathrm{TM}}$, Ocean Nutrition Canada, Ltd., Bedford, NS, Canada); refined menhaden oil (Omega Pure ${ }^{\mathrm{TM}}$, Omega Protein, Reedsville, VA) and a 70 purified marine oil (ROPUFA 30 ${ }^{\mathrm{TM}}$ n-3 Food Oil, Roche Lipid Technologies, Derbyshire, DE). The fish oil concentrate (Table 1) contained 89.5\% omega-3 FA and mixed tocopherols; the refined menhaden oil

72 contained 26.5\% omega-3 FA and a combination of mixed tocopherols and tertiary butylhydroxyquinone (TBHQ); and the purified marine oil contained 26.8\% omega-3 FA and mixed

74 tocopherols, ascorbyl palmitate, and a rosemary extract. These oils were added at levels of either $1.5 \%$ .J. Agric. Food Chem. 2004;52(26):7944-7949 
or $2.5 \%(w / w)$. As each of the fish oils contained different concentrations of omega-3 fatty acids, each

76 level of addition was adjusted to result in the same level (i.e. 1.5\% or 2.5\%) omega-3 fatty acids added to each gel. The lower level is roughly equivalent to that naturally occurring in salmon flesh (9-10) and 78 the higher level was designed to test the maximum levels achievable without a loss of the physical and sensory characteristics of the surimi gels in comparison to control.

80 Prepared surimi pastes were extruded into stainless steel tubes (22.7 cm length, $1.9 \mathrm{~cm}$ i.d.) using a sausage stuffer (The Sausage Maker, Buffalo, NY). A two-step thermal treatment was used. Samples

82 were first cooked in a $90{ }^{\circ} \mathrm{C}$ bath for 6 min to allow gelation of surimi, roughly simulating rapid cooking achieved by continuous sheet cooking in industry. Samples were held at this temperature for 8 min., 84 removed from the tubes, and placed in a double vacuum plastic bag with low permeability to oxygen (Cryovac, Duncan, SC). A second heat treatment in a $85{ }^{\circ} \mathrm{C}$ bath for $15 \mathrm{~min}$ was then applied in 86 simulation of industrial pasteurization practices (12). Samples were then immediately cooled in an ice/water slush and subsequently held below $4{ }^{\circ} \mathrm{C}$ for up to two months. Chemical, physical and sensory analyses were conducted initially (after overnight storage) and at one month intervals.

Sensory evaluation. A six-member trained sensory panel performed descriptive sensory (flavor, odor)

90 analyses. Aroma, flavor, and after-taste notes were determined using a fourteen-point scale: not detectable (0-2), threshold (2-5), slight (6-9), moderate (10-13), and strong (14). The panel was initially 92 trained $(13,14)$ to evaluate flavor changes in fish gels and were retrained in this study to score crab product attributes with emphasis on recognizing fishy odor and aroma as these are the two negative 94 sensory attributes most often associated with the addition of omega-3 FA. Control gels containing no added oil and stored at $-50{ }^{\circ} \mathrm{C}$ were used for reference. Each sensory property was expressed as average 96 of the data from all each panelists.

Lipid Stability. Production of lipid oxidation volatiles was determined by the manual solid phase 98 microextraction (SPME) technique for low molecular weight oxidation compounds (15). A SPME extractor containing a $75 \mu \mathrm{m}$ filter of Carboxen ${ }^{\mathrm{TM}}$ polydimethylsiloxane (Supelco, Bellefonte, PA) was 100 used. Two (2) g of chopped gel were placed in a $12 \mathrm{~mL}$ vial (Tekmar Dohrmann Co., Cincinnati), J. Agric. Food Chem. 2004;52(26):7944-7949 
flushed with nitrogen, closed with a headspace clamp, and kept in chilled storage at $4{ }^{\circ} \mathrm{C}$ for up to 2

months. Headspace was sampled at 0, 1, and 2 months. Oxidation volatiles present in surimi gels were extracted onto SPME fibers by heating vials at $60{ }^{\circ} \mathrm{C}$ for 15 min with the manual SPME apparatus in the 104 injection mode. Desorbtion of volatiles from the fiber onto the GC column occurred by heating the SPME apparatus in the injection mode of the GC injection port for 2 min at $180{ }^{\circ} \mathrm{C}$ with a carrier gas flow rate of $2.0 \mathrm{~mL} / \mathrm{min}$. An HP-5 capillary column (30 m x $0.25 \mathrm{~mm}$ i.d., $0.25 \mu \mathrm{m}$ film thickness, Hewlett Packard, Palo Alto, CA) was used for separation. Volatiles were identified by comparison to authentic standards followed by confirmation with a GC-mass spectrometry system consisting of a HP 5890 II GC and HP 5972 mass selective detector (Hewlett-Packard, Avondale, PA). The GC oven temperature was cryogenically cooled and programmed from $-20{ }^{\circ} \mathrm{C}$ to $160{ }^{\circ} \mathrm{C}$ at $15^{\circ} \mathrm{C} / \mathrm{min}$ with initial and final hold times of 4.5 and 1 min, respectively. The oven temperature was programmed for an 112 increase of $40{ }^{\circ} \mathrm{C} / \mathrm{min}$ from $160{ }^{\circ} \mathrm{C}$ to $220{ }^{\circ} \mathrm{C}$ and a final hold time of $1.5 \mathrm{~min}$. The carrier gas was electronically controlled at a linear velocity of $1.5 \mathrm{~mL} / \mathrm{min}$. The ionization voltage for the mass spectrometer was $70 \mathrm{eV}$. The mass scanning range was 35-350 KDa. Two replications were performed.

The polyene index (ratio of polyunsaturated (PUFA) to saturated (SAT) fatty acids) was calculated

116 from the gas chromatographic data. As DHA (C22:6) represents the most highly unsaturated and most labile fatty acid present in fish oils, the loss of DHA relative to palmitic acid (C16:0) is often used as a sensitive index indicative of the oxidation of PUFA, and was therefore used in the present study (16-17). It was expressed as the percentage of polyene index relative to the polyene index of the raw oil.

120 To determine the fatty acid composition of each oil and of omega-3 enriched gels, total crude lipids were extracted by a modification of the Bligh and Dyer (18) procedure in which $0.001 \mathrm{~g} / \mathrm{L}$ of BHT was 122 added to a chloroform / methanol solvent system (1:2), followed by extraction, cleanup, and subsequent drying of lipids. The fatty acid methyl esters (FAME) were prepared by the addition of 2 mL boron 124 trifluoride per 25 mg of lipid. Crude FAME were washed with distilled water, extracted twice with 2 $\mathrm{mL}$ hexane, and dried under nitrogen at $35{ }^{\circ} \mathrm{C}$ (19). Crude FAME were purified by thin layer 126 chromatography using a solvent elution system consisting of petroleum ether/diethyl ether (95:5), .J. Agric. Food Chem. 2004;52(26):7944-7949 
followed by drying under nitrogen. Purified FAME were re-dissolved in iso-octane for injection. The

128 FAME were analyzed on an HP model 5890A GC (Avondale, PA), equipped with a flame ionization detector, an HP 3393 integrator and a Dell computer, Model D1028L (Round Rock, TX) for data storage 130 and handling. The GC contained a DB 225, $30 \mathrm{~m} \mathrm{x} 0.25 \mathrm{~mm}$ fused silica column (J\&W Scientific Co, Folsom, CA), that was temperature programmed from $140{ }^{\circ} \mathrm{C}$ to $200{ }^{\circ} \mathrm{C}$ at a rate of $1.7^{\circ} \mathrm{C} / \mathrm{min}$ with 132 both injector and detector temperatures set at $260^{\circ} \mathrm{C}$. Identification of FAME was based on comparisons of retention times of test samples to methyl ester standards (462 and 68D, NuChek Prep, Elysian, MN)

134 using C21:0 as the internal standard. Absolute response factors were calculated for each identified fatty acid peak using the normalization technique described by Sampugna et al. (20). Fatty acid composition 136 was expressed as weight percent of total FAME and was determined in duplicate.

Gel Color Analysis. Color differences between treatment gels and the control were determined by 138 reflectance on a Chroma Meter CR 300 (Minolta Camera Co., Ltd., Osaka, Japan) using the CIE Lab scale $\left(\mathrm{D} 65 / 10^{\circ}\right)$, where $\mathrm{L}^{*}$ (black 0 to light 100 ), a* (red 60 to green -60 ) and $\mathrm{b}^{*}$ (yellow 60 to blue 140 60) were used to measure lightness, redness, and yellowness. Whiteness Index was defined as: 100$\left[\left(100-\mathrm{L}^{*}\right)^{2}+\mathrm{a}^{2}+\mathrm{b}^{*}\right]^{1 / 2}$. The result was expressed as the average of six samples.

142 Gel Fracture Properties. Gel samples were tempered to ambient temperature $\left(20{ }^{\circ} \mathrm{C}\right)$ prior to analysis. Gels were cut into 29-mm long cylinders, each end of which was glued to plastic disks using 144 cyanoacrylate glue. The cylinders were ground on a Precision Machine (Model GC-TG92 US, Gel Consultants, Inc., Raleigh, NC) and formed into capstan shapes with a center diameter of 10 mm. Gels 146 were vertically mounted and twisted to fracture at $2.5 \mathrm{rpm}$ on a Hamann Torsion Gelometer (Gel Consultants Inc., Raleigh NC) to determine stress (strength) and strain (deformability) at fracture 148 displacements as described by Diehl et al. (21). Standard deviations on measurements among replicates (6 samples) were less than $10 \%$.

150 Statistical Analysis. One-way analysis of variance (ANOVA) was carried out using the SPSS system (SPSS Inc., Chicago, Illinois). Significant differences between pairs were determined by Bonferroni test. 152 In the case of sensorial test, significant difference between means was resolved by means of confidence .J. Agric. Food Chem. 2004;52(26):7944-7949 
intervals using non parametric statistics by the Kruskal-Wallis test using the SPSS system (SPSS Inc.,

154 Chicago, Illinois). Level of significance was set at $\mathrm{p} \leq 0.05$.

\section{$156 \quad$ RESULTS AND DISCUSSION}

158 Sensory Testing. Food product palatability is one of the main sensory characteristics limiting the level of fortification with omega-3. Results of this study showed that $2.5 \% \mathrm{n}-3$ was the highest level 160 omega-3's that was acceptable for the sensory panel (taste and texture) (data not shown). The faint and fish-like odor was masked by the added crab flavor aroma extracts allowing the use of a significant level (2.5\%) of fish oil. These findings agree with those of Kolanowski et al. (4), in which they demonstrated that high flavor intensity products are more effective at masking the slightly fishy taste and odor 164 associated with the addition of omega-3 fatty acids.

Figure 1 shows the profile of the descriptive sensory attributes of control surimi gels with no oil added. However it should be noted that the additions of the crab flavor resulted in noticeable crab aroma-flavor and sweetness notes in the crab analogues. Crab gels formulated with fish oil concentrate

(Figures 2 and 3) contained significantly higher intensity of fishy attributes including taste, aftertaste (AFT) and aroma than present within the control (Fig.1). For treatments containing fish oil concentrate, the sensory attributes reached the rejection level of off-flavors and aroma at both levels of addition (i.e $1.5 \%$ \& 2.5\%) after one month of storage. Thus, treatments containing fish oil concentrate were not evaluated for further sensory analyses. These results show that although the strong crab flavor was effective in masking moderate fishy taste and aroma attributes, the fish oil concentrate contained an especially high concentration of fishy sensory notes that would not allow its use in a typical processed seafood product, such as a crab-flavored analog. 


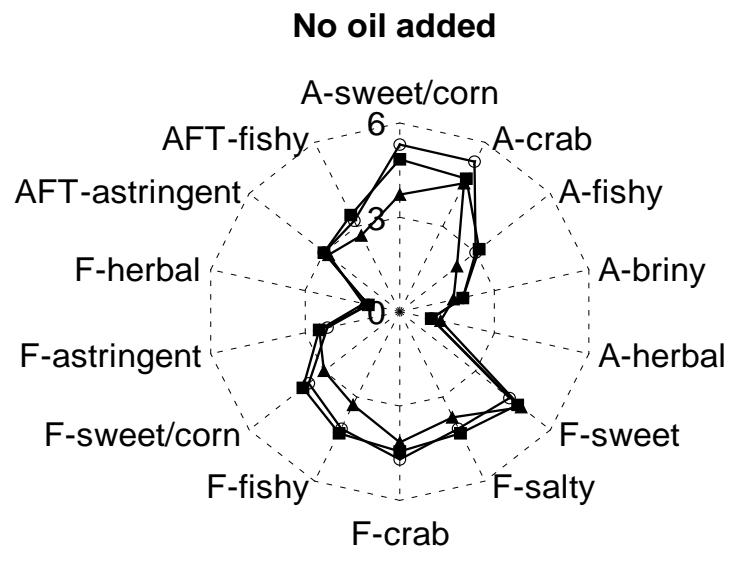

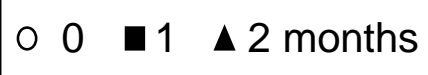

178 Figure 1. Change in sensory attributes of surimi gels containing no added omega-3 fatty acids. $\mathrm{A}=$ aroma, $\mathrm{F}=$ flavor, $\mathrm{AFT}=$ aftertaste

Fish oil concentrate

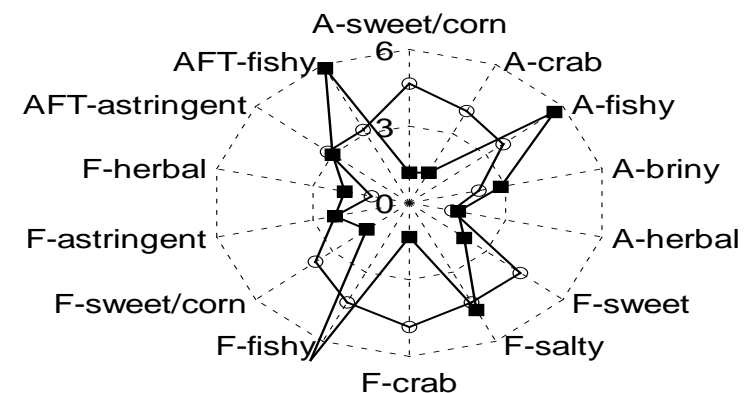

Menhaden oil

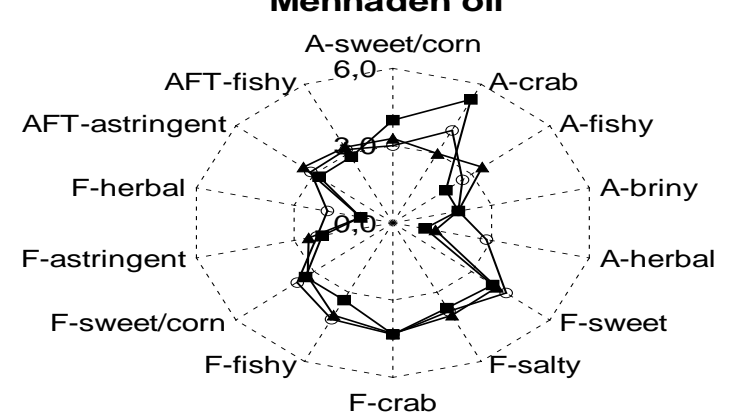

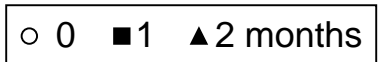

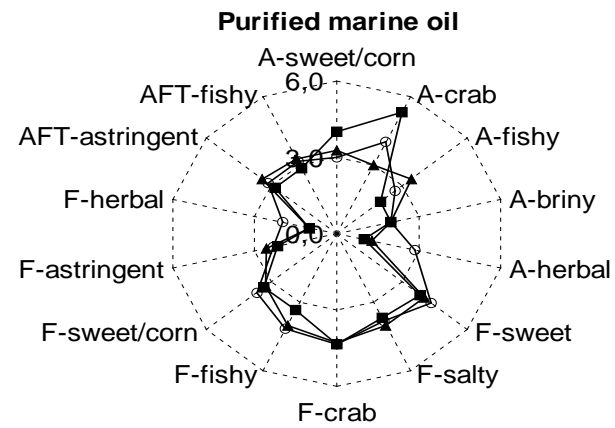

180

Figure 2. Change in sensory attributes of surimi gels fortified at $1.5 \%$ omega- $3 . \mathrm{A}=$ aroma, $\mathrm{F}=$ flavor 182 and AFT: = aftertaste 
Fish oil concentrate

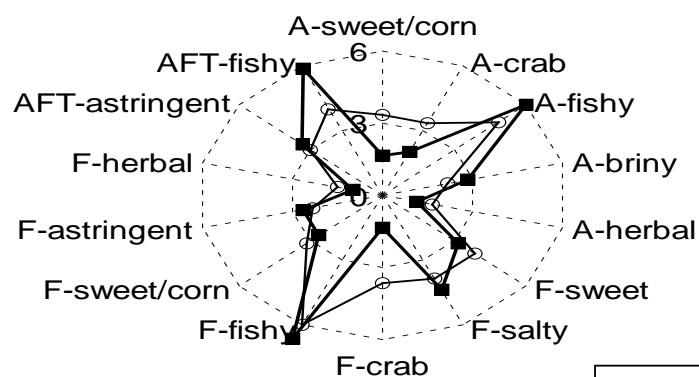

$0 \quad 0 \quad \square 1 \quad \Delta 2$ months
Menhaden oil

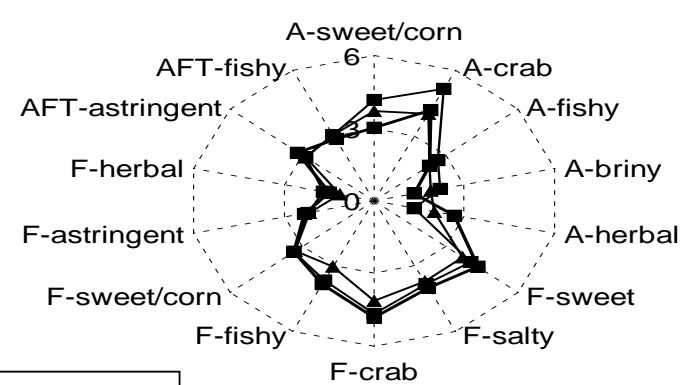

\section{Purified marine oil}

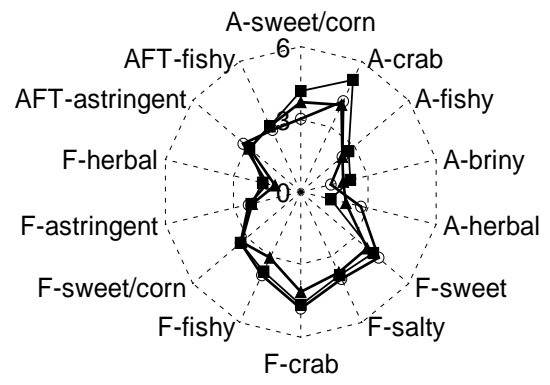

Figure 3. Change in sensory attributes of surimi gels fortified at $2.5 \%$ omega-3. $A=$ aroma, $F=$ flavor and AFT $=$ aftertaste

Treatments containing menhaden oil and purified marine oil (Figures 2 and 3) showed sensory profiles more similar to those of the control gel containing no added oil. Even though the sweet/corn flavor that was observed in the control samples was not present in treatments containing menhaden and purified marine oils, the added crab flavor and aroma were effective in masking trace amounts of offflavors that may have resulted from addition of the omega-3 fish oils. Other studies in which menhaden 194 oil was used in cookies formulations showed that when the cookies were stored at room temperature, fish oil-containing cookies reached the rejection level of off-flavor intensity (mildly oxidized off-flavor) at ten days whereas treatments containing added ascorbic acid reached an additional seven days of shelf 
life (6). The author explained that the combination of food ingredients and an oven-baking process caused menhaden oil to be more vulnerable toward oxidative degradation.

Lipid Stability. Formation of volatile compounds was similar in all samples. Among 10-15 peaks 200 detected, the major compounds positively identified were acetaldehyde, ethanol, dimethyl disulfide, ethyl acetate, butanediol, and hexanoic acid. As volatile concentrations did not increase significantly 202 during storage whereas ethanol was used as a carrier for the crab flavors added, it was concluded that the extent of oxidation during the 2 month storage period was not a significant factor in decreasing sensory 204 attributes. Immediately following elaboration of gels, minor concentrations of hexanal was also observed. However, as hexanal is a major oxidation product of omega-6 oxidation and appeared as a 206 minor peak in control samples and decreased with storage, it was concluded that it too was not a major contributing factor to changes in sensory profiles or in lipid stability.

208 The processing of fish oils to remove unwanted odors and off-flavors has also been improved considerably with some studies (6) showing that during the refining of fish oil, the process removes 210 almost all compounds related to fishy odors. However during storage, additional odorous compounds such as pentylfuran and aldehydes can be generated. These types of compounds cause green and beany 212 odors whereas the presence of ketones create metallic-type off-flavors. The presence of decatrienal and heptenal has been shown to impart fishy odor to foods. The production of volatiles is also influenced by 214 the degree of unsaturation of the oil. One such study (22) identified unsaturated aldehydes and ketones containing two or three double bonds that were highly correlated with the loss of DHA during storage of 216 fish oil. High correlation between both hexanal and pentanal levels as determined by SPME/GC and lipid oxidation as measured by 2-thiobarbituric reactive have been observed in other oxidation studies

218 (15). Studies of beef gels stored under chilled conditions found increased concentrations of total aldehydes, hydrocarbons and alcohols due to storage time at $3{ }^{\circ} \mathrm{C}$ where as total ketones varied due to 220 time only (23).

Thus, the combined effects of short storage time in this study (i.e. 2 months), chilled storage 222 conditions, and the presence of volatile oxidation products below the level of detection may all have .J. Agric. Food Chem. 2004;52(26):7944-7949 
contributed to the absence of significant oxidation products being measured in this study. The recent

224 availability of more highly stabilized fish oils containing a variety of antioxidants may also have played a significant role in reducing the degree of oxidation and the lack of measurable oxidation products. All 226 of the oils used in this study had been stabilized with a combination of commercial antioxidants and natural antioxidants that may well have contributed to the absence of off-flavors and odors observed 228 during chilled storage.

Fatty acid composition. Figure 4 shows a comparison of the change in the polyene index in 230 relationship to the composition of the raw oils from each one of the three sources of added fish oil. Due to the heat treatment, there was a decrease in polyene index values at different percentages depending of 232 type of oil added. A 15-20 \% decrease occurred in samples made with fish oil concentrate and also menhaden oil and only 5-10\% occurred in purified marine oil. In all cases, there was a tendency of 234 higher losses when using higher amounts of omega-3 fatty acids, though none of the losses between treatments were significant. However, after two months of chilled storage, the percentage of loss was 236 similar in menhaden and purified marine oil samples (about 25-30 \%). Therefore, with the exception of oils lost during the initial cooking operations, all of the treatments maintained approximately $75 \%$ of 238 polyene index of the raw oil at the end of the storage. 


\section{Fish oil concentrate}
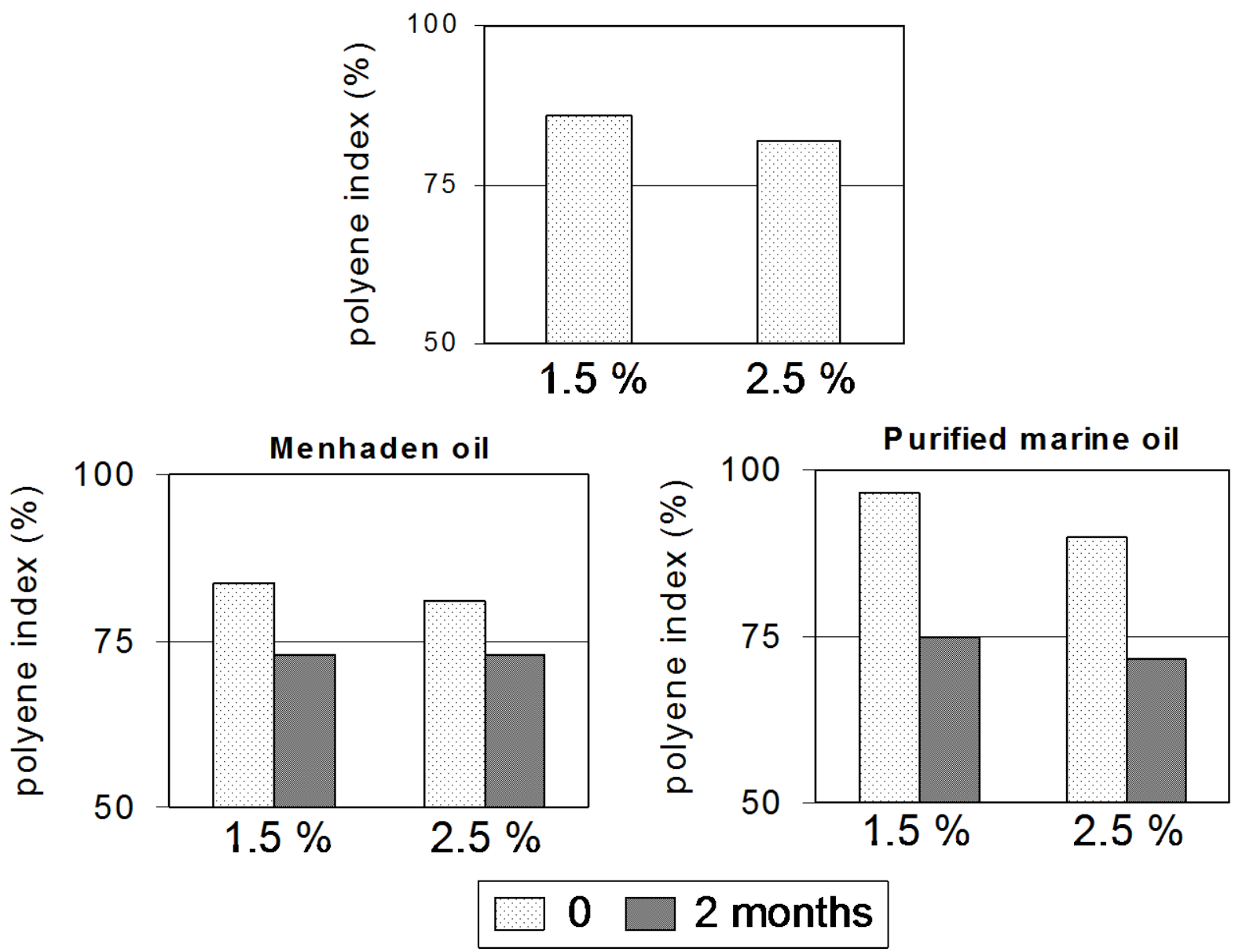

Figure 4. Change in polyene index (ratio C22:6/C16:0) of surimi gels after cooking (bars) and after two 242 months of chilled storage (solid).

Ghazala, Aucoin and Alkanani (24) studied the stability of fatty acids after pasteurization and found 246 that more EPA and DHA are retained when pasteurization process is done at a low temperature $\left(65^{\circ} \mathrm{C}\right)$ compared to a higher temperature. Differences in the inherent composition of the commercial oils (Table 1) and their antioxidants may also have played a role. For example, the fish oil concentrate contained the highest level of omega-3's, and was the only oil in which only mixed tocopherols were 250 used as the only type of antioxidant added. While tocopherols are regarded as excellent antioxidants, 
their effectiveness as the sole antioxidant in fish oils has not been equivalent to that of combinations of 252 natural and synthetic antioxidants, especially TBHQ. Both the menhaden oil and the purified fish oil contained lower levels of omega-3's and a variety of natural and synthetic antioxidants that could have 254 contributed to a greater degree of stability observed in these two oils compared to the fish oil concentrate. The purified marine oil contained added rosemary which may have contributed to the 256 higher protective effect in this relatively short term study although its effectiveness in long-term study has been questioned. Similar studies (25) have reported that the addition of rosemary extract may offer

258 limited extension of storage stability because of its involvement in the initial free radical reactions.

260 Gel Color. Only minor changes in color occurred during chilled storage (Figure 5). Initially the control gel (no oil added) was less opaque, and significantly $(\mathrm{p} \leq 0.05)$ lower in whiteness index than 262 those fortified with oils. The increased whiteness can be attributed to the increase in lightness ( $\mathrm{L}^{*}$ values) caused by the dispersion of light resulting from the presence of emulsified oil droplets. Since 264 whiteness of color is a desirable attribute in surimi seafood products, the addition of oil only enhanced the quality of the product. During chilled storage, there was a tendency toward increased whiteness with 266 increased storage time with most of the samples showing significant $(p \leq 0.05)$ increases after the first month of chilled storage. 

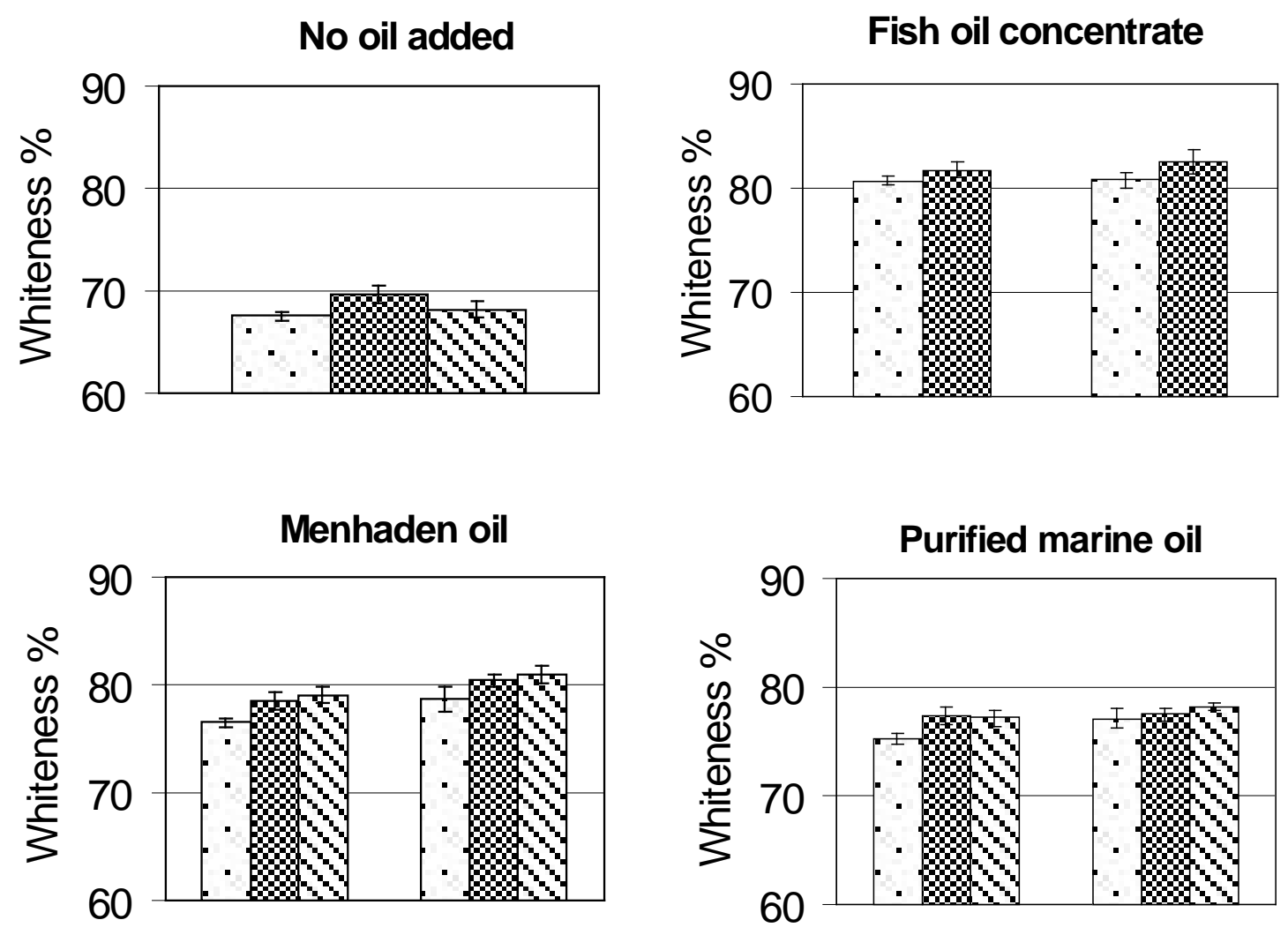

\section{$\square 0 \otimes 1$ \ 2 months}

270 Figure 5. Change in whiteness of surimi gels containing the different oils and levels of omega-3. Error bars represent the standard deviation of six samples.

Gel Fracture Properties. There was a significant increase in shear stress of gels containing menhaden

274 oil and purified marine oil at both omega-3 concentrations at the beginning of storage in comparison with control with no oil added. This was expected because of the added oil substituted for the same

276 amount of water used in the formulation (Figure 6). No increase shear stress occurred in the case of fish oil concentrate since it contained the highest level of omega-3 FA and required the smallest amount of 278 oil (about 2-3 \%) to obtain the desired level. Though oil as an ingredient can modify the characteristics of the gels, no major modifications were obtained since the formulation contained starch and egg white. 280 These results agree with a similar study carried out by Pérez-Mateos et al (26) in which increased 
breaking force and hardness were observed in heat-induced mackerel gels containing omega-3 oil added at the $2.5 \%$ level compared to gels containing no added oil.
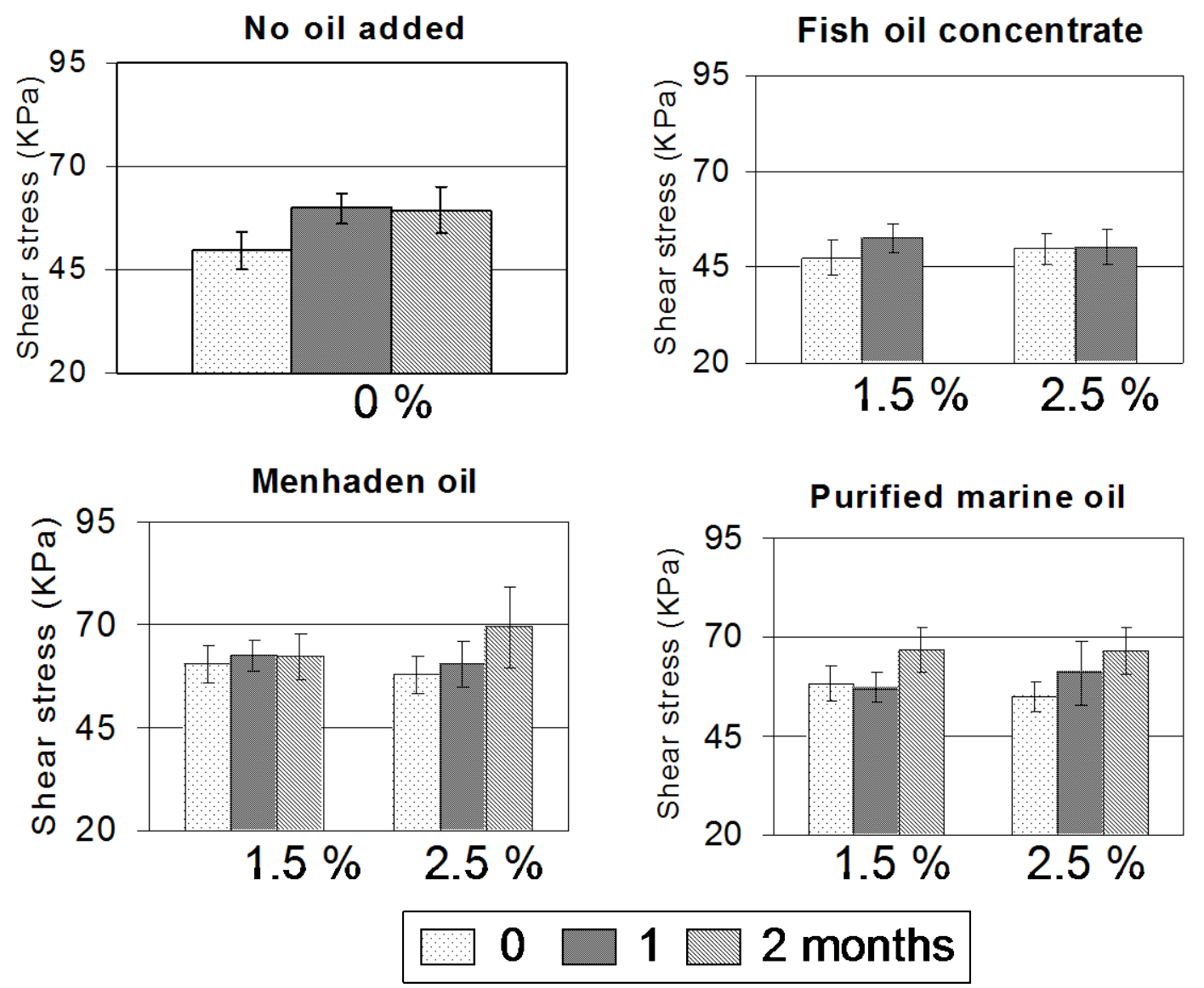

Figure 6. Changes in shear stress values of surimi gels containing the different oils and levels of omega-

3. Error bars represent the standard deviation of 6 samples.

Stress (strength) increased in gels with no oil added during the first month of storage $(\mathrm{p} \leq 0.05)$ as would be expected due to bond realignment in the first weeks (Figure 6) and a similar tendency to increase with storage time was observed in samples containing added oil, though not significant in that cases. 
Shear strain values (Figure 7) did not decrease with the addition of oil and no significant differences

294 where found due to the type of oil used since the water content was adjusted depending on the amount of oil added. Shear strain values remained stable throughout the storage in all the samples.
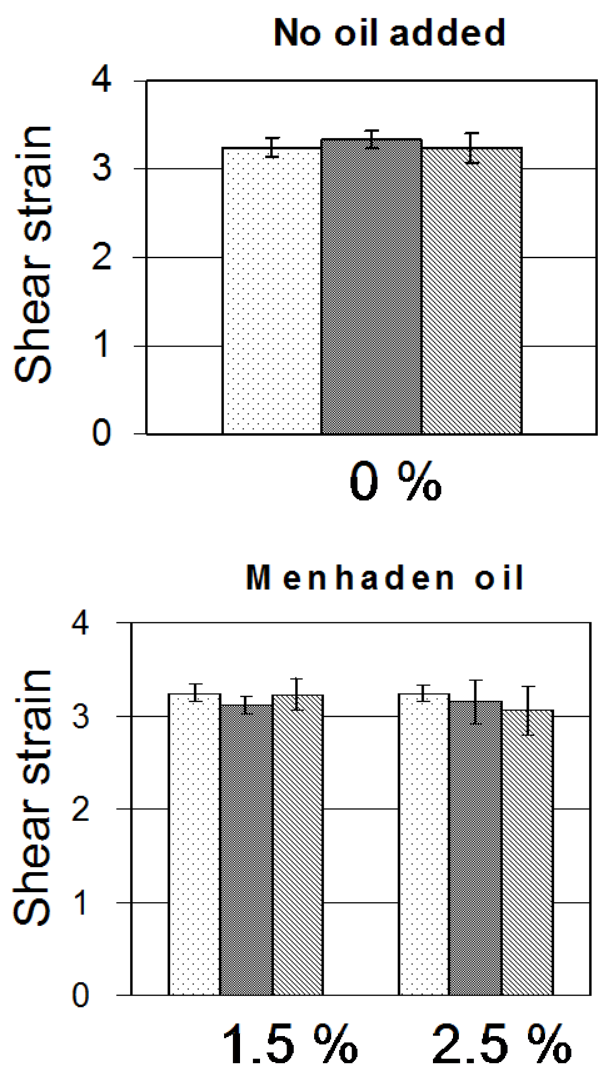
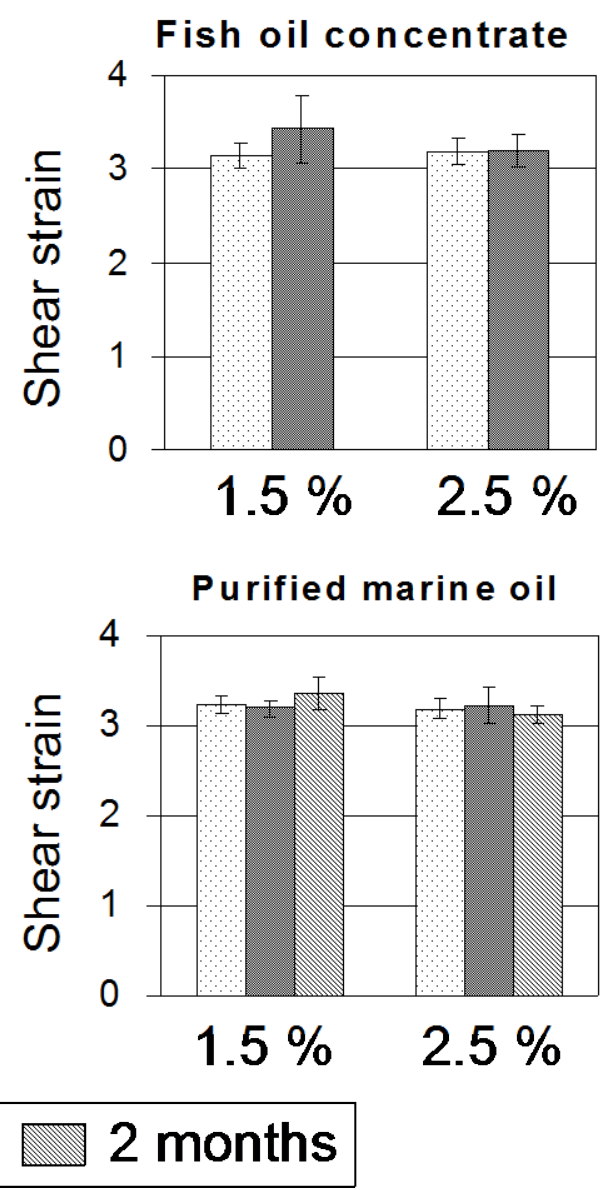

Figure 7. Changes in shear strain values of surimi gels containing the different oils and levels of omega3. Error bars represent the standard deviation of 6 samples.

\section{CONCLUSIONS.}

302 Fortification of surimi-based crab analog products to contain omega-3 FA levels equal or exceeding that of natural seafood products appear quite feasible. The sensory and analytical data showed that the type of fish oil used and its antioxidant system can be critical to avoiding off-flavors and aromas that can distract from consumer acceptance of such products. Fish oils can be added to attain levels of 2.5\% 
gels.

\section{ACKNOWLEDGMENT}

Paper number FSR-04- 02 of the Journal series of the Department of Food Science. The research was 310 funded (in part) by the North Carolina Agricultural Research Service of the College of Agricultural and Life Sciences and the use of trade names does not imply endorsement, nor criticism of similar ones not

312 mentioned. Additional support is acknowledged from the University of North Carolina Sea Grant College Program and the National Fishery Institute. Dr. Miriam Pérez-Mateos is grateful to the Spanish

314 Ministry of Education, Culture and Sport (MECD) - Fulbright Program for the postdoctoral fellowship that sponsored her.

Material consist of one table and seven figures of data and one mathematical formula describing how to

318 Table 1. Summary of fatty acids compositions of fish oil concentrate, Menhaden oil, and purified marine oil expressed as percentage composition of total fatty acids identified.

\begin{tabular}{|c|c|c|c|}
\hline $\begin{array}{l}\text { Major classes of } \\
\text { fatty acids }\end{array}$ & Fish Oil Concentrate & Menhaden Oil & Purified Marine Oil \\
\hline Saturates & 2.0 & 41.5 & 38.5 \\
\hline Monoenes & 6.1 & 34.0 & 31.4 \\
\hline PUFA $^{(a)}$ & 91.9 & 24.5 & 30.1 \\
\hline n-3 PUFA ${ }^{(b)}$ & 89.5 & 21.5 & 26.8 \\
\hline
\end{tabular}

(a) PUFA = total polyunsaturated fatty acids; (b) total n-3 polyunsaturated fatty acids 


\section{REFERENCES}

324 1. Calder, P.C. Immunomodulatory and anti-inflammatory effects of omega-3 polyunsaturated fatty acids. Proc. Nutr. Soc. 1996, 55,737-774.

326 2. Newton, I.S. Global food fortification perspectives of long-chain $\omega 3$ fatty acids. In The return of $\omega 3$ fatty acids into the food supply, I. Land-based animal food products and their health effects. World

328 Rev Nutr Diet; Simopoulos, A.P., Ed.; Karger: New York, NY, 1998; vol 83, pp199-209.

3. Chapman K.W.; Regenstein J. M. Use of fish oils in food products. In Seafood safety, processing and biotechnology; Shahidi, F., Jones, Y., Kitts, D., Eds.; Technomic Pub. Co., Inc.: New York, NY, 1997, chapter 16, pp151-159.

4. Kolanowski, W.; Swiderski, F.; Berger, S. Possibilities of fish oil application for food products enrichment with $\omega$-3 PUFA. Int. J. Food Sci. Nutr. 1999, 50, 39-49.

5. Newton, I.; Snyder, D. Nutritional aspects of long-chain omega-3 fatty acids and their use in bread enrichment. Cereals Foods World 1997, 42(3), 126-131.

6. Lin C. F. Flavor chemistry of fish oil. In Lipids in food flavors; Ho, C.T, Hartman, T.G, Eds.; ACS Symposium series: Washington, DC, 1994; chapter 558, pp208-232.

7. Rudolph, M.J.; Huang, Y.; Garretson-Lowry, S.; Lobo, G.P. Fortification of reduced fat ice cream with omega-3 long chain polyunsaturated fatty acids. Presented at the IFT Annual Meeting: New Orleans, LA, 2001; June 23-27.Abstract \#29-7.

8. Lanier, T.C; Martin, R.E.; Bimbo, A.P. Nutritional implications of increased consumption of engineered seafoods. Food Technol. 1988, (May), 162-165.

9. Bell, J.G.; McEvoy, J.; Webster, J.L.; McGhee, F.; Millar, R.M.; Sargent, J.R. Flesh lipid and carotenoid composition of Scottish farmed Atlantic salmon (Salmo salar). J Agric. Food Chem. 1998, 46, 119-127. 
11. AOAC. Official Methods of Analysis, 14 ed. Assoc. of Official Analytical Chemists, 1984, Arlington, VA.

12. Shie, J.S.; Park, J.W. Physical characteristics of surimi seafood as affected by thermal processing conditions. J. Food Sci. 1999, 64 (2) 287-290.

13. Stone, J.; Siedel, J.; Oliver, S.; Woosley, A. Sensory evaluation by quantitative descriptive analysis. Food Technol. 1974, 28,24-34

14. Henry, L.K.; Boyd, L.C.; Green, D.P. The effects of Croprotectants on the sensory Properties of Frozen Blue Crab (Callinectes sapidus) Meat. J. Sci. Food Agric. 1995, 69, 21-26.

15. Brunton, N.P.; Cronin, D.A; Monahan, F.J.; Durcan, R.A. Comparison of solid-phase microextraction (SPME) fibers for measurement of hexanal and pentanal in cooked turkey. Food Chem. 2000, 68, 339-345.

16. Boyd, L.C.; King, M.C.; Sheldon, B.A. Rapid Method for Determining the Oxidation of n-3 Fatty Acids. J. Am. Oil Chem. Soc. 1992, 69, 325-330.

17. King, M.F.; Boyd, L.C.; Sheldon, B.W. Antioxidant properties of individual phospholipids in a salmon oil model system. J. Am. Oil Chem. Soc. 1992, 69(6), 545-551.

18. Bligh, E.G.; Dyer, W.J. A rapid method of total lipid extraction and purification. Can. J. Biochem. 364 Physiol. 1959, 37, 911-914.

19. Metcalfe, L.D.; Schmitz, A.A. The rapid preparation of fatty acid esters for gas chromatographic 366 analysis. Anal. Chem. 1961, 33, 363-368.

20. Sampugna, J.; Pallansch, L.A.; Enig, M.G.; Keeney, M. Rapid analysis of trans fatty acids on SP368 2340 capillary columns. J. Chromatogr. 1982, 249: 245-255.

21. Diehl, K.C.; Hamann, D.D.; Whitfield, J.K. Structural failure in selected raw fruits and vegetables. J. Tex. Studies 1980, 10 (4), 371-400.

22. Lee, H.I; Lee, Y.; Yang, J.Y; Kizito, S. A.; Weese, J.S. Oxidation of DHA-enriched fish oil and extraction and identification of the volatile oxidation products. Presented at the IFT Annual Meeting: New Orleans, LA. 2001, June 23-27, Abstract \#30A-35. 
374 23. Butler, A.J.; Larrick, D.K. Effect of antioxidants on the sensory characteristics and storage stability of aseptically processed low-fat beef gels. Meat Sci. 1993, 35, 355-369.

376 24. Ghazala, S.; Aucoin, J.; Alkanani, T. Pasteurization effect on fatty acid stability in a sous vide product containing seal meat (Phoca groenlandica). J. Food Sci. 1996, 61(3), 520-523.

378 25. Langourieux, S.; Escher, F.E. Sulfurous off-flavor formation and lipid oxidation in heat-sterilized meat in trays. J. Food Sci. 1998, 63 (4), 716-720.

380 26. Pérez-Mateos, M.; Gómez-Guillén, M.C.; Hurtado, J.L.; Solas, T.; Montero, P. The effect of rosemary extract and omega-3 unsaturated fatty acids on the properties of gels made from the flesh 382 of mackerel (Scomber scombrus) by high pressure and heat treatments. Food Chem. 2002, 79(1), 18. 